\section{§4. Effects of Time-varying ExB Flow on Slab Ion-temperature-gradient Turbulence}

Maeyama, S. (Tokyo Institute of Technology), Ishizawa, A., Watanabe, T.-H., Škorić, M.M., Nakajima, N.,

Tsuji-Iio, S., Tsutsui, H. (Tokyo Institute of Technology)

Effects of time-varying sheared $\mathrm{E} \times \mathrm{B}$ flow on turbulence driven by slab ion temperature gradient instabilities are investigated by means of Landau fluid simulation. Here, the $\mathrm{E} \times \mathrm{B}$ flow, which consists of stationary and time-periodic oscillatory parts, is externally imposed to the turbulence. The dependence on the amplitude and frequency of $\mathrm{E} \times \mathrm{B}$ flow is examined in the case that the amplitude of oscillatory part is the same or less than that of stationary part. The ion heat transport caused by turbulence oscillates with the same period as the $\mathrm{E} \times \mathrm{B}$ flow and the time-averaged transport coefficient is larger than the coefficient which is evaluated without the oscillatory part. The time-averaged coefficient is maximized when the amplitude of oscillatory part is equal to that of stationary part. As the frequency of $\mathrm{E} \times \mathrm{B}$ flow increases, the time-averaged coefficient decreases and is close to the coefficient which is evaluated without the oscillatory part. This mechanism is explained by introducing a kind of the logistic equation which describes the time evolution of transport coefficient as a response of turbulence to the amplitude of $\mathrm{E} \times \mathrm{B}$ flow ${ }^{1)}$.

Nonlinear Landau fluid simulations of the ITG turbulence with the externally imposed sheared $\mathrm{E} \times \mathrm{B}$ flow were performed to investigate the effects of time-varying shear flow on transport suppression. The $\mathrm{E} \times \mathrm{B}$ flow is externally imposed to the turbulence, and its amplitude is given by Aext $(\mathrm{t})=\mathrm{As}+\mathrm{Ao} \sin (\omega \mathrm{ot})$. We considered the case where the energy of $\mathrm{E} \times \mathrm{B}$ flow is close to or larger than that of the turbulence, and examined the dependence of the turbulent heat transport on the amplitude and frequency of $\mathrm{E} \times \mathrm{B}$ flow. Figure 1 shows the time-averaged transport coefficients against the amplitude of oscillatory part normalized to the stationary one, Ao/As, for different As. As As increases, the time-averaged coefficient decreases since the stronger shear flow is more effective to distort eddies of ITG turbulence. On the other hand, as Ao increases for a fixed As, the time-averaged coefficient rises for As $>$ Ao, but drops for As $<$ Ao. Thus, the time-averaged coefficient has extremal values when As $=$ Ao. The timeaveraged transport coefficient is plotted in Fig. 2 as a function of the frequency of $\mathrm{E} \times \mathrm{B}$ flow. The dotted line shows the transport coefficient for the stationary $\mathrm{E} \times \mathrm{B}$ flow case. The solid line represents the analytic solution of model equation, which is discussed in the next section. The time-averaged transport coefficient decreases as the frequency of $\mathrm{E} \times \mathrm{B}$ flow increases, and becomes close to the transport coefficient for the stationary $\mathrm{E} \times \mathrm{B}$ flow case. This indicates that the effect of the oscillation on the timeaveraged decreases as the frequency increases.

In summary, it was found that the time-averaged transport coefficient is larger than the transport coefficient in case without oscillation. This implies that the oscillation of $\mathrm{E} \times \mathrm{B}$ flow enhances the transport. This transport enhancement is attributed to the response of turbulence, as the turbulence grows up when $|\operatorname{Aext}(\mathrm{t})|$ is low but decays when $|\operatorname{Aext}(\mathrm{t})|$ is high. The temporal variation in the transport coefficient is impulsively peaked (not sinusoidal), and hence, its time average does not vanish so that the coefficient is enhanced. The enhancement in the transport is found in the cases with As>Ao. Furthermore, for larger Ao, the transport is maximized when As = Ao (Fig. 1). This is because the turbulence is enhanced when the oscillatory part cancels out the stationary one, and leads to the weak flow shear. Next, we investigated the frequency dependence of the heat transport. As the frequency of $\mathrm{E} \times \mathrm{B}$ flow increases, the effect of oscillation on heat transport is weakened, and the transport coefficient is close to the value for the case without oscillation. This is because the turbulence follows to the time-varying $\mathrm{E} \times \mathrm{B}$ flow with the delay, which is characterized by the typical time scale of turbulence such as the growth rate, and cannot keep up with the high frequency oscillation of $\mathrm{E} \times \mathrm{B}$ flow. To mimic the observed properties of the transport coefficient, which has the time delay and impulsive enhancement in its evolution, we introduced the logistic-type equation, where a target value of transport coefficient was supposed to be determined by the instantaneous value of $\mathrm{E} \times \mathrm{B}$ flow.

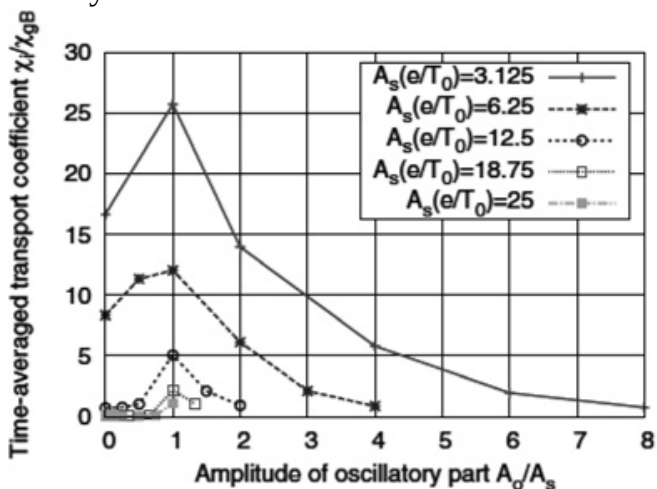

Fig. 1. Time-averaged transport coefficient as a function of the amplitude ratio.

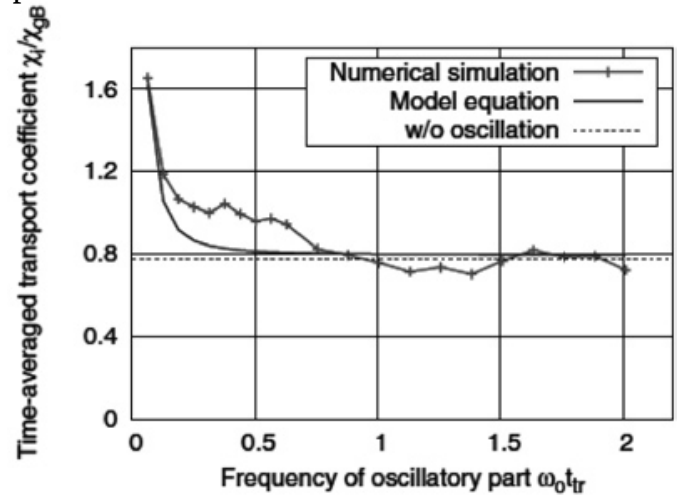

Fig. 2. Time-averaged transport coefficient as a function of the frequency of the ExB flow. As a reference, the transport coefficient evaluated without oscillation is plotted.

1) Maeyama, S, Ishizawa, A., Watanabe, T.-H., Škorić, M. M., Nakajima, N., Tsuji-Iio, S., Tsutsui, H., Physics of Plasmas 49, (2010) 062305. 